Revue interdisciplinaire sur la Grèce archaïque

\title{
Autochtonies grecques : la terre, la mère, le genre
}

Greek Autoch thonies: The Soil, the Mother, the Gender

Jean Alaux et Françoise Létoublon

\section{(2) OpenEdition}

\section{Journals}

Édition électronique

URL : https://journals.openedition.org/gaia/2530

DOI : 10.4000/gaia.2530

ISSN : 2275-4776

Éditeur

UGA Éditions/Université Grenoble Alpes

Édition imprimée

ISBN : 978-2-37747-292-5

ISSN : $1287-3349$

Référence électronique

Jean Alaux et Françoise Létoublon, « Autochtonies grecques : la terre, la mère, le genre », Gaia [En

ligne], 24 | 2021, mis en ligne le 31 mai 2021, consulté le 09 décembre 2021. URL : http://

journals.openedition.org/gaia/2530; DOI : https://doi.org/10.4000/gaia.2530

Ce document a été généré automatiquement le 9 décembre 2021.

Gaia. Revue interdisciplinaire sur la Grèce archaïque 


\title{
Autochtonies grecques : la terre, la mère, le genre
}

\author{
Greek Autochthonies: The Soil, the Mother, the Gender
}

Jean Alaux et Françoise Létoublon

\section{Remerciements}

Nous avons une grande gratitude envers les éditeurs de cet hommage à notre ami Pellizer, que nous avons rencontrés autour de lui à Grenoble et à Trieste, ainsi qu'envers les rapporteurs anonymes de la revue qui ont enrichi considérablement notre bibliographie et la problématique.

1 Une première version de cette recherche a été présentée oralement à un colloque franco-polonais sur les représentations de la terre organisé à Paris par une collègue comparatiste et publié à cette occasion ${ }^{1}$. Il nous a semblé que cette mise à jour avec une problématique enrichie par la question linguistique de la relation entre genre (angl. gender) et genre grammatical et par les nombreuses publications intervenues depuis lors serait opportune dans un recueil d'hommage à Ezio Pellizer, qui a organisé à Trieste en juin 2009 une belle rencontre en mémoire de Nicole Loraux, centrée sur le thème de la femme ${ }^{2}$.

Revenir sur l'ensemble des mythes grecs d'autochtonie en tenant compte de leurs logiques singulières, de leurs variantes et de leurs nombreuses implications politiques et anthropologiques serait souhaitable, mais paraît impossible dans le cadre limité d'un article. La question est difficile et a donné lieu à de nombreuses controverses. Pour le colloque franco-polonais, nous nous sommes décidés à travailler à deux ${ }^{3}$, en partant de nos travaux respectifs et avec la facilité que nous offrait alors l'université de Grenoble où nous étions collègues, pour essayer d'analyser le débat assez vif qui a opposé Nicole Loraux et Marcel Detienne. Les pièces du débat sont d'un côté Les enfants d'Athéna (1990) et Né de la terre (1996), de l'autre Comment être autochtone? (2003)4. La thèse de Nicole Loraux peut être résumée comme suit: la naissance du premier Athénien, issu de la terre même, puis élevé par la déesse Athéna, éponyme de la cité, permet aux Athéniens de se poser face aux autres cités grecques comme les seuls à ne pas être «venus

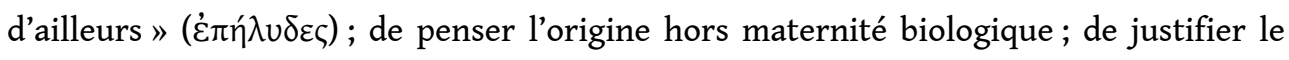


fait qu'à Athènes, les hommes mâles seuls sont citoyens, participant de plein exercice à tous les domaines de la vie publique; d'éclairer enfin le paradoxe selon lequel la cité d'Athènes est la seule des cités grecques à porter le nom d'une déesse, Athéna. Les Athéniens sont ainsi à la fois fils de la terre et nourris par une déesse vierge.

3 S'agissant du rapport à la terre, de l'origine, et tout particulièrement de celle des hommes, la mythologie grecque frappe en effet par la multiplicité et l'enchevêtrement des différents récits qu'elle a produits ${ }^{5}$, eux-mêmes disséminés dans des sources variées (littéraires, épigraphiques), parfois lacunaires, qu'il faudrait à leur tour confronter aux témoignages archéologiques et iconographiques.

On sera donc conduit à opérer un choix dans cette vaste matière. Celui qui s'impose à nous dans le cadre actuel (en 2005 comme aujourd'hui) a déjà été privilégié par les textes fondateurs qui ont marqué la tradition occidentale, et qui mettent à notre disposition le matériau le plus parlant : c'est dire qu'Athènes et les œuvres qu'elle a produites occupent une place centrale dans notre réflexion ${ }^{6}$.

C'est là, en effet, que l'autochtonie joue un rôle majeur dans la représentation de soi construite par la cité. Quelques rappels plus larges et plus généraux s'imposent toutefois d'abord.

Il faut reconnaitre avec Cynthia Patterson que le texte de saint Augustin qui rapporte l'exclusion des femmes athéniennes de la citoyenneté ne fait pas partie du corpus des mythes anciens connus dans l'Athènes classique ${ }^{7}$. Mais il nous semble que l'on peut adopter une position plus bienveillante envers Loraux, celle que défend Violaine Sebillotte-Cuchet en 2012 dans les lignes qu'elle lui consacre dans "Touchée par le féminisme », en particulier p. 156-157 :

Les mythes sont saisis par Nicole Loraux comme des discours construits, réélaborés à partir de matériaux narratifs plus anciens, par une cité qui les «travaille " pour leur faire exprimer son propre «imaginaire», voire ses "fantasmes». Dans le premier chapitre des Enfants d'Athéna, Nicole Loraux analyse les mythes athéniens de l'autochtonie comme les variantes d'une même structure désignée par le nom de «mythe civique », de " représentation de la cité », ou d' "imaginaire civique ». Cette structure révèle un argument majeur [...] : l'exclusion des femmes de la cité, voire leur négation ${ }^{8}$.

7 Si l'on accepte cette interprétation, on voit que le passage de la Cité de Dieu peut s'intégrer dans l'ensemble des mythes athéniens d'autochtonie comme variante: on note d'ailleurs qu'il s'agit chez Augustin de citer un auteur plus ancien, le mythologue $\mathrm{du} \mathrm{I}^{\mathrm{er}}$ siècle avant J.-C., Varron, qui joue sur les différentes versions des mythes et s'amuse parfois à en créer d'autres : pour Patterson, il s'agit d'un artefact tardif, qui n'a rien à voir avec les représentations de l'époque classique, mais dans l'autre hypothèse, il peut s'agir d'une variante à partir des traditions anciennes sur laquelle Varron a brodé sans inventer totalement ${ }^{9}$.

\section{La terre, la mère et l'origine des hommes}

Pour la pensée grecque, la terre dans son rapport avec l'origine se présente sous la double espèce de la "glèbe » et de la "glaise ", selon qu'elle se fait divinité créatrice, lieu de naissance ou matière à fabrication ${ }^{10}$. Sans nous attarder sur ce dernier aspect, rappelons que, chez Hésiode, c'est bien avec de l'eau mêlée de terre ${ }^{11}$ que l'artisan Héphaïstos façonne ce simulacre, ce fatal artifice qu'est la première femme (nommée 
Pandora dans les Travaux), d'où sera issue, dans un étonnant engendrement du même par le même, la "race des femmes" (Théogonie, 591-592). Remarquons que, dans les poèmes hésiodiques, la création de la première femme constitue la punition que Zeus inflige aux hommes trop favorisés par Prométhée ${ }^{12}$ : les hommes mâles semblent alors déjà présents ${ }^{13}$.

Du côté de la théogonie hésiodique, l'une des trois entités primitives se nomme Gaia, à partir de laquelle la mise en place de l'univers s'effectue en l'espace de quatre générations : fécondée par Ciel, qu'elle a conçu seule en même temps que Flot $^{14}$, elle enfante une série de Titans ${ }^{15}$, dont Kronos, le dernier-né. Pendant deux générations, une rivalité de pouvoir oppose alors un père et un fils ; Kronos mutile par la ruse son père Ouranos, qui refuse de laisser advenir à la lumière les enfants que Gaia met au monde; Kronos, uni à sa sœur, engendre une série de divinités, dont Zeus, lui aussi le dernier-né. Zeus à son tour se rebellera contre son père dévorateur : grâce à la ruse, il échappe à l'avidité paternelle et, aidé d'autres puissances, mène contre son père et les Titans une longue guerre victorieuse ${ }^{16}$ qui les relègue aux marges de l'univers, dans les tréfonds du Tartare. Tout est prêt pour qu'il engendre, avec sa sœur Héra ou d'autres, la deuxième génération des Olympiens. Mais aucune mention des humains...

Si l'on considère à présent les mythes d'origine des hommes, le premier trait caractéristique, quelque peu embarrassant pour qui, comme nous, voudrait qu'une lumière simple présidât aux commencements, est la complexité des débuts, faite de «faux départs et de vraies fins ${ }^{17}$ ", de délais et de redoublements, la plupart du temps dans un contexte de querelle divine; on en donnera ici quelques exemples.

11 À propos du genre humain tout entier, on mentionnera le mythe de Deucalion, fils de Prométhée, et de Pyrrha, fille d'Épiméthée et de Pandore : Zeus a noyé dans le déluge les hommes de la race de bronze; Deucalion et Pyrrha sont seuls épargnés; abordant sur une terre ferme, ils y jettent chacun à leur tour des pierres; de celles que jeta l'époux naquirent les hommes, et de celles que jeta l'épouse, les femmes; mais Deucalion et Pyrrha eurent aussi, dit-on, des enfants, dont Hellen, l'ancêtre des Grecs ${ }^{18}$. On rattachera à ce thème la mention, proverbiale dès l'Iliade, des hommes «nés des pierres ou des arbres ».

12 Si l'on examine à présent l'univers des cités, on citera le cas d'Argos, où le premier humain, Phoroneus, né du dieu fleuve Inachos (et parfois de son union avec la nymphe Mélia ${ }^{19}$ ), se heurte au mauvais vouloir de Poséidon, parce qu'il lui préfère Héra ; le dieu provoque alors l'assèchement mortel des terres; il faudra attendre de nombreuses générations pour que Poséidon se réconcilie avec la race d'Inachos en s'unissant à Amymoné et en restaurant pour la cité l'eau et la vie ${ }^{20}$.

On n'oubliera pas non plus le mythe arcadien, auquel les Athéniens se réfèrent souvent comme à un contre-exemple ${ }^{21}$ : il y eut d'abord Pélasgos, l'autochtone ${ }^{22}$, dont le fils Lykaon, pour avoir offensé Zeus, devient loup et revient à la sauvagerie ; c'est à sa fille Kallistô qu'il revient alors de perpétuer la lignée: unie à Zeus, elle enfante Arkas, éponyme de l'Arcadie, qui pouvait enfin commencer ${ }^{23}$. Elle-même est métamorphosée en ourse.

14 On mentionnera aussi l'unique étymologie possible pour le nom de Pélée, le père d'Achille, qui est un nom grec de la "boue ", $\pi \eta \lambda$ ó $^{24}$, ce qui pourrait faire de lui un homme fait de terre à la manière d'Adam. 

collectives propres à chaque culture ${ }^{31}$. En linguistique, on distingue en général le genre grammatical motivé qui correspond au sexe, certaines langues qui n'ont pas de neutre l'introduisant dans la flexion pronominale (français il / elle / ça), et l'on tend à attribuer le genre des autres noms à l'arbitraire du signe linguistique. Mais l'originalité de Meillet a été de chercher, au-delà de cette notion saussurienne d'arbitraire, un fondement à la catégorie du genre dans les cas où elle n'est pas déterminée par le sexe, et d'appliquer à cela les principes de la méthode comparative à l'élaboration de laquelle il a beaucoup participé. Il a ainsi pu montrer en s'appuyant sur le fait de la flexion nominale que l'opposition essentielle en indo-européen n'est pas entre masculin et féminin mais entre animé et inanimé. À l'intérieur du genre animé, les formes du masculin ont la primauté, et les formes spécifiques de féminin, quand elles apparaissent, sont en général dérivées à partir de lui. Il y a une relation privilégiée entre les notions d'animé et de personne, d'inanimé et de non-personne, suivant la terminologie bien postérieure de Benveniste. La comparaison des différents systèmes des genres du hittite, de l'arménien, du celtique, du grec, etc., aboutit à une conclusion générale sur l'évolution des langues indo-européennes et sur leur chronologie. Sous l'influence de la sociologie et de l'anthropologie de son temps (Durkheim, Mauss, Lévy-Bruhl, entre autres), Meillet affirme en 1931 que le genre est une « affaire de conception, non de grammaire ${ }^{32} »$. Dans un article important de 1920, Meillet propose une série de noms désignant des réalités dont le genre grammatical parait arbitraire pour des mentalités modernes : eau, feu, noms d'astres, soleil, étoile, lune, sommeil, songe, jour, nuit, parties du corps (pied, main, bras, langue, foie, cœur, os, rate, coude, genou), pour lesquels la reconstruction comparative montre des éléments intéressants, surtout 
quand les termes sont très archaïques. Ces éléments sont désignés par des neutres dans certaines langues (ainsi pour « eau » et «feu » le grec ǔ $\delta \omega \rho / \pi \tilde{u} \rho$ ), mais par des animés dans d'autres, et dans ce cas leur genre semble "complémentaire», masculin / féminin : latin ignis / unda ou aqua, français feu / eau, etc. De même pour le soleil et la

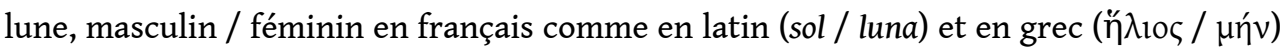
ou inversement comme en allemand (der Mond "lune», masc. / die Sonne "soleil», féminin), ce qui permet de penser que les genres correspondaient à d'anciennes conceptions mythologisantes de cette complémentarité.

L'article de Meillet ne cite pas les noms du ciel et de la terre, qui semblent pourtant correspondre parfaitement à sa théorie: les langues indo-européennes conçoivent en effet très régulièrement le ciel comme masculin et la terre comme féminin, ce qui correspond aux conceptions mythologiques de la terre comme mère nourricière et du ciel qui la féconde ; le sanskrit a connu un dieu Ciel, dont le nom masculin dyauh est surtout utilisé dans le composé (au duel) dyava-prthivi « ciel et terre », et dont le second terme est en fait un adjectif féminin substantivé signifiant « la large ». Le dieu Varuna paraît être une autre forme divinisée du ciel. La terre a aussi d'autres noms, tous

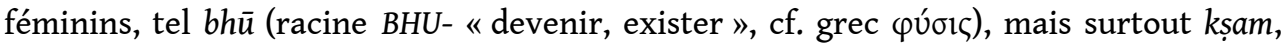
parallèle au grec $\chi \theta \omega \hat{v}$, permettant avec d'autres témoignages tels que hittite tekan, latin humus, homo, entre autres, une reconstruction sous la forme *ghedhom/ ghdhom ${ }^{-33}$. La diversité des différents témoignages dans les langues indo-européennes ne permet certes pas de reconstituer linguistiquement un couple indo-européen terreciel originel, mais la constance des genres grammaticaux fait penser que cette conception a existé, et que son incarnation dans la Théogonie d'Hésiode sous la forme de Gaia et Ouranos correspond tout à fait à l'imaginaire linguistique qu'ont eu les IndoEuropéens d'une terre-mère.

Les travaux récents sur le genre n'ont guère réfléchi à ces questions linguistiques, mais on peut pourtant citer Sebillotte-Cuchet (2007b, 237-239), qui étudie la notion d'« idéalité civique » à partir du texte du serment des éphèbes athéniens que conserve une inscription publiée par Robert en 1938, en reprenant le terme à Loraux $(1981,299)$ :

Ce qui féminise la patris, c'est incontestablement la terre qui, en grec, se dit très souvent au féminin $(\dot{\eta} \chi \theta \dot{\omega} v, \dot{\eta} \chi \omega ́ p \alpha, \dot{\eta} \gamma \tilde{\eta})^{34}$. Or, en ce cas, le féminin renvoie incontestablement à une fonction, celle de la mère comme lieu de production de

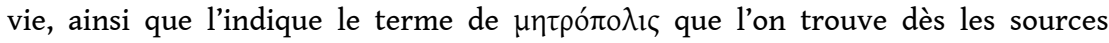
classiques pour désigner la cité d'origine. Platon a précisément décrit cette fonction dans le Timée qui envisage la production de l'univers. Le lieu, troisième catégorie après le modèle et la copie du modèle, est comparé à une mère dans la mesure où il est «réceptacle» (ं்

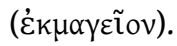

Ainsi, pour l'imaginaire grec, la terre est très nettement caractérisée par son genre féminin qui en fait une entité productive et nourricière.

21 Le genre grammatical est un point de vue partiel, que nous avons développé parce qu'il s'agit d'un moyen généralement ignoré d'accéder aux représentations. Mais l'imaginaire de l'autochtonie lié à la fécondité de la terre se laisse analyser dans maints textes grecs: dans son article de 2018, le spécialiste de Platon qu'est Arnaud Macé

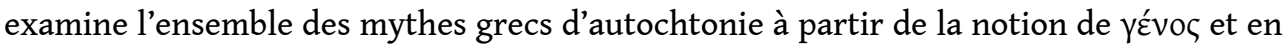
dégage l'usage politique dans une perspective proche de celle de Loraux, en utilisant outre le Ménexène, le mythe de l'Atlantide dans le Critias et un passage de la République : 
Le mythe, dans ses diverses variantes, suggère qu'un peuple pourrait être directement sorti de sa terre, né de son sol, d'une manière qui établisse entre son territoire et lui une intimité telle qu'elle scelle son destin (p. 19) [...] il ne s'agit plus de réclamer l'autochtonie relative d'un indigénat (nous venons d'ici aussi longtemps que nous puissions remonter) mais d'affirmer être tout simplement les premiers occupants de cette terre, et de n'être jamais venu [sic] d'ailleurs (p. 24).

\section{Les mythes athéniens d'autochtonie et leur récurrence : Cécrops, Érichthonios, Érechthée}

À Athènes même, l'origine autochtone s'y prend plus qu'à deux fois ${ }^{35}$, sur fond de querelle récurrente entre Poséidon et Athéna, future déesse poliade : il y eut Cécrops, le premier autochtone, mi-homme, mi-serpent ${ }^{36}$, qui rassembla les hommes en cité, institua le mariage et même, comme Detienne ne manque pas de le rappeler ${ }^{37}$, fit qu'hommes et femmes délibérèrent ensemble ; les femmes, qui avaient la majorité en cette aurore, votèrent pour Athéna, pourtant peu encline à incarner la féminité ; Poséidon frustré inonda l'Attique; les femmes, dit saint Augustin citant Varron, perdirent le droit de vote, ne s'appelèrent plus Athéniennes, et les enfants cessèrent de porter le nom de leurs mères ${ }^{38}$. Les temps historiques pouvaient commencer.

Deuxième acte, le plus cher, sans nul doute, au cœur des Athéniens : sur le sol de l'Acropole, Héphaïstos poursuit de son désir la déesse vierge Athéna; elle l'évite et, selon certaines versions, essuie le sperme du dieu d'un brin de laine ${ }^{39}$, qu'elle jette à terre : du sol ainsi fécondé naît le premier Athénien, Érichthonios ${ }^{40}$, qu'Athéna toute à la joie de n'avoir pas été mère, soulève dans ses bras nourriciers.

Mais Érichthonios a un doublet, Érechthée, dont on le fait parfois aussi le fils ou petit-fils (par Pandion interposé) ${ }^{41}$ : lors de la guerre contre Eumolpos, descendant de Poséidon, roi d'Éleusis, il doit sacrifier sa fille ou ses filles pour qu'Athènes l'emporte. C'est en son honneur que fut édifié sur l'Acropole l'Érechthéion, au ve siècle ${ }^{42}$.

Entre Cécrops et Érichthonios se glisse à l'occasion Cranaos ${ }^{43}$, roi d'Athènes, qui tantôt transmet le pouvoir à Érichthonios, tantôt en est le grand-père, et dont l'épouse se nomme Praxithéa, comme celle d'Érechthée (un deuxième Cécrops se retrouve d'ailleurs comme fils d'Érechthée et de Praxithéa II ${ }^{44}$.

Être les seuls de tous les peuples, grecs ou non, à n'être pas issus d'un immigrant (ع́ $\pi \eta \lambda u \varsigma^{45}$ ), mais de la terre même, c'est ce dont au plus haut point se glorifient les Athéniens, et que répètent les oraisons funèbres ${ }^{46}:$ cette origine commune, sans cesse réactualisée dans le cadre de cérémonies civiques permet aussi de fonder l'égalité entre tous les citoyens, dotés de surcroît du prestige aristocratique qui s'attache aux

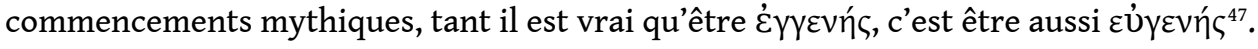

L'autre avantage, toujours placé sous le signe de l'engendrement du même par le même, est l'exclusion des femmes impliquée par la substitution de la terre à la mère dans la naissance d'Érichthonios, qu'il convient de mettre en rapport avec le vieux

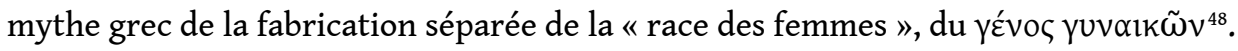

À cet égard, les gestes d'Athéna, évitant la semence d'Héphaïstos, la détournant vers le sol pour ensuite jouer le rôle de la nourrice de l'enfant qui en est issu, prennent une importance symbolique majeure. Si l'on considère les documents iconographiques les plus célèbres ${ }^{49}$, la représentation de la naissance d'Érichthonios se concentre en effet sur trois personnages centraux : Gê, plus ou moins émergée du sol, qui tend l'enfant ; 
Érichthonios lui-même, tout élan vers la déesse poliade; Athéna enfin, qui le reçoit pour l'élever ${ }^{50}$.

Enfin, d'un point de vue politique, la substitution de la terre à la mère permet de légitimer le sacrifice du citoyen à la guerre. La croyance est aussi une créance. Elle est même un gain : en mourant pour la cité, le soldat échange une vie éphémère pour une gloire immortelle et rend à la terre la vie qu'elle lui a donnée. "Bénéfices de l'autochtonie » eût dit N. Loraux, car l'imaginaire lui aussi (lui surtout, peut-être) spécule et capitalise...

\section{L'autochtonie et le genre tragique : Athènes face à Thèbes}

30 La cité d'Athènes ne s'est pas contentée de célébrer son propre mythe d'autochtonie dans sa version privilégiée, celle de la naissance d'Érichthonios; pour conjurer peut-être la part d'exclusion qu'elle implique et les contradictions latentes que celui-ci porte en lui (toujours la même question : comment articuler l'origine topique et la reproduction sexuée, la répétition du même et la succession des générations engendrées par l'union des deux sexes ?), elle a construit son négatif, qu'elle donne à voir sur la scène du théâtre de Dionysos, des Sept contre Thèbes d'Eschyle (467 av. J.-C.) aux Bacchantes d'Euripide (405 av. J.-C.), en passant par l'Antigone et l'Ædipe Roi de Sophocle. Cette anti-Athènes, née d'une mauvaise autochtonie, s'appelle Thèbes ${ }^{51}$ : Thèbes, pourtant fondée par le "Phénicien » Cadmos, devient alors la cité où le même se replie mortellement sur soi, sur fond de meurtres intestins, d'incestes, de cumul des liens de parenté.

31 Tout part des Spartes et tend à y retourner, même si leur autochtonie est à proprement parler «dérivée » puisqu'ils furent "semés" par $\mathrm{Cadmos}^{52}$. Mais la logique de l'imaginaire n'est pas simple, surtout quand elle est travaillée par l'idéologie : il faut que les Spartes se soient mathématiquement entretués sitôt apparus; mais il faut également qu'en demeurent quelques-uns pour que puisse être intégré à la généalogie thébaine le principe qu'ils incarnent (notamment Chthonios, grand-père de Nyctéis, mère de Labdacos, et Échion, époux d'Agavé, père de Penthée et ancêtre de Créon).

De ce trait témoigne évidemment la figure tragique des fils d'ÆEdipe, Étéocle et Polynice, qui, héritiers du faix paternel, n'ont d'autre vocation que d'imprégner la terre de leurs sangs mutuels, dans une manière de consanguinité à la fois originelle et fictive. Le texte des Sept contre Thèbes d'Eschyle s'achève sur ce retour à la terre des deux sangs mêlés par le meurtre: "Leur haine a cessé: dans la terre où a coulé leur sang ( $(\hat{o} \mu \alpha \iota \mu o l)^{53}$.»

33 En s'entretuant, les fils d'œEdipe réitèrent le geste des Spartes, pour qui naissance et mort, meurtre et suicide, se télescopent au rebours de toute généalogie visible. Il y a plus : dans la mesure où un tel geste, qui aurait dû interdire toute descendance s'il n'y avait eu celle de Cadmos et d'Harmonie, se trouve répété au terme de la lignée commune, les fils d'Ædipe réactivent la négativité fondamentale, et même en un sens, fondatrice, de Thèbes. C'est alors toute l'histoire des Labdacides qui se lit comme la mise en scène d'une anti-généalogie, sur fond d'inceste, de confusion des liens et d'abolition du temps. 
dans la pièce d'Euripide: Dionysos, né à Thèbes de la fille de Cadmos, Sémélé, est le fruit d'une double gestation, celle qu'il connut dans le ventre de sa mère, humaine, et celle que lui assura son père divin Zeus lorsque, Sémélé foudroyée, il cousit et couva l'enfant dans sa cuisse ${ }^{55}$. En ce qui concerne Penthée, la paternité d'Échion, le Sparte, revient avec insistance dans le texte d'Euripide et doit être mise en relation avec les traits tyranniques propres à Penthée, qui le rapprochent de l'Étéocle des Sept contre Thèbes d'Eschyle ${ }^{56}$. Quel meilleur moyen, pour la tragédie, de suggérer que l'origine et la croyance autochtones ne sont jamais innocentes? athénienne se construise sur l'exclusion politique du féminin: c'est le cas de M. Detienne, dans une polémique qui l'opposa à N. Loraux dès la parution des Enfants d'Athéna et qui se poursuivit pendant plusieurs années ${ }^{57}$. L'argumentation repose en majeure partie sur l'interprétation des fragments de l'Érechthée d'Euripide ${ }^{58}$.

Restons donc dans l'univers de la tragédie grecque et revenons-en à l'histoire de la guerre d'Athènes contre Eumolpos, roi d'Éleusis : seul un sacrifice peut sauver la cité ; ce sera celui de la fille (ou des fille ${ }^{59}$ ) d'Érechthée, dont la mère, Praxithéa, exalte la valeur dans un grand discours patriotique. Victoire donc, pour Athènes, mais Poséidon, d'un coup de son trident, enfouit Érechthée dans le sol d'Athènes ${ }^{60}$. Praxithéa, «celle qui œuvre pour la déesse » (Athéna) devient la première prêtresse d'Athéna Polias, ancêtre rituelle de cette Lysimaché dont S. Georgoudi a évoqué l'histoire, totalement confondue avec sa fonction ${ }^{61}$. On s'en tiendra à quelques remarques, inspirées par la lecture des fragments d'Euripide à la lumière de ce que nous savons des jeux de mise à distance et de brouillage propres à la tragédie : car la tirade de Praxithéa pourrait être une ironique parodie des topoi de l'oraison funèbre, dont Platon s'est peut-être souvenu en plaçant dans la bouche d'Aspasie le discours du Ménexène.

On retrouve, dans cette tirade, l'opposition, poussée à l'extrême, entre les Athéniens, purs autochtones (fr. 14,8) et les autres cités, « formées d'éléments importés de toute origine » (v. 10). Il est d'ailleurs révélateur que le citoyen venu d'ailleurs soit comparé à une « méchante cheville fichée dans une poutre » (v. 12) : l'expression employée, $\dot{\alpha} \rho \mu$ ò $\varsigma$ 


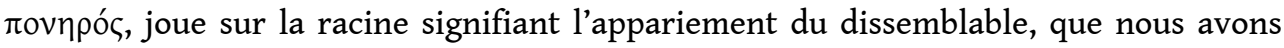
rencontrée, à Thèbes, dans le nom d'Harmonie ${ }^{62}$.

Si Praxithéa vante sans réserve le sacrifice d'un individu pour sauver la cité, elle n'en souligne pas moins que seule l'absence d'un rejeton mâle la conduit à souhaiter le dévouement de sa fille (22-27) ${ }^{63}$. Il est intéressant également de noter que cette fille

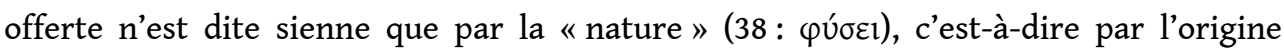
(nous comprenons que sa destinée finale appartient à la cité), mais c'est bien aussi comme un fait de «nature " qu'au début de sa tirade l'autochtonie est présentée (8:

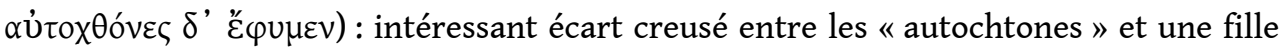
née d'une mère dont on rappellera que, contrairement à son époux Érechthée, elle n'est pas autochtone elle-même (comment une femme pourrait-elle l'être ?) ${ }^{64}$.

41 Un autre effet, révélateur de la distorsion tragique, surgit à la lecture des fragments. Dans sa tirade patriotique, Praxithéa réprouve les « larmes maternelles » (fr. 14, 28-30), qui affaiblissent les enfants en partance pour la guerre (on songe bien sûr au contrôle sévère exercé sur le deuil féminin des funérailles célébrées au Céramique ${ }^{65}$ ). Mais, dans la suite de l'action, c'est probablement la même Praxithéa qui se lamente avec le chœur dans un de ces chants de plainte où l'on perçoit sans doute le mieux la singularité de la voix tragique ${ }^{66}:$ «Combien il est rebelle aux larmes et cruel, celui qui ne gémit pas sur nos malheurs! » s'écrie-t-elle alors (fr. 22, v. 44) ${ }^{67}$.

La tragédie, instrument critique de l'idéologie autochtone ? Comment en douter? Mais elle n'est pas seule à l'être: on a déjà mentionné Platon et la stratégie du Ménexène. Cette stratégie ne joue pas seulement au plan des motifs et des topoi. Elle traverse la complexité de la langue même, la syntaxe, le rapport entre comparant et comparé, entre signifiant et signifié, lorsque, par exemple, il y est dit, au terme d'un développement sur la "vraie mère » que fut de tout temps la terre, que « ce n'est pas la terre qui a imité la femme dans la conception et l'enfantement, mais la femme la terre » (237e-238a). Proposition mise sans hésitation au compte de la "pensée grecque " par bien des Modernes. Or, dans cette phrase, comme l'a montré avec force N. Loraux, l'ordre métaphorique donne à voir sa propre subversion - car il s'agit bien d'une dénégation, énoncée comme telle par l'ironie platonicienne : le signifiant générationnel rend possible l'assimilation de la terre à la femme, et donc la fiction d'une imitation de la première par la seconde, dans un beau continuum de sonorités "galactiques " (oủ

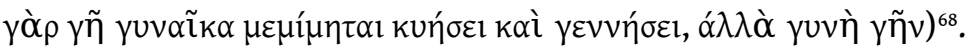

43 La question de l'altérité apparait donc centrale à la fois dans les discours produits par les Grecs sur l'autochtonie et dans ceux que tiennent, à partir d'eux, les Modernes. Elle fait retour au cœur même des constructions qui veulent l'éluder. Le perpetuum mobile des temps primordiaux ${ }^{69}$, volontiers transféré sur les autochtonies autres qu'athéniennes, témoigne de cette vibration consubstantielle à l'origine. D'un côté, une loi clairement perçue, celle du mouvement des peuples, des arrivées successives, des va-et-vient; de l'autre, le besoin d'exclure de la terre d'Athènes ce trouble que, pourtant, l'on sait originel. Mais, curieusement, ce même mouvement de va-et-vient se reporte du coup sur l'axe temporel, puisque origine et présent se recréent mutuellement dans une double vectorisation qui vise à annuler la durée. Ce qui était, dans l'ordre spatial, mouvements successifs et jamais clos, devient, dans une fiction temporelle beaucoup plus rassurante, parcours circulaire entre un début et une suite sans cesse réactualisée sur le mode du même ${ }^{70}$. Le texte tragique, on l'a vu, est là pour exacerber les tensions sous-jacentes à une telle opération. 

terre ne remplacera complètement la mère, même si jamais la tentation ne cessera de recouvrir l'une par l'autre ${ }^{72}$.

\section{BIBLIOGRAPHIE}

ALAUX Jean, « Ordre et désordre en territoire grec (VIII ${ }^{\mathrm{e}}-\mathrm{IV}^{\mathrm{e}}$ siècles av. J.-C.) », dans J. Chevallier, J. Alaux, B. Piettre et al. (éd.), Désordre(s), Paris, Presses universitaires de France, 1997a, p. 17-28.

ALAUX Jean, « Entre répétition et différenciation : genos et phusis autochtones à Athènes », RHR, 214 (4), 1997b, p. 467-480.

ALAUX Jean, «Compte rendu de Laurent Gourmelen, Kékrops, le Roi-Serpent. Imaginaire athénien, représentation de l'humain et de l'animalité en Grèce ancienne, Paris, Les Belles Lettres, 2004 ", Gaia, 10, 2006, p. 331-333.

BOEHRINGER Sandra \& SEBILLOTTE-CUCHET Violaine, « Vingt ans de réflexion. Mètis et le genre (1992-2012) », dans S. Boehringer \& V. Sebillotte-Cuchet (dir.), Mètis, hors série, Des femmes en action. L'individu et la fonction en Grèce antique, Paris / Athènes, Éditions de l'EHESS, 2013, p. 5-18.

BOEHRINGER Sandra \& SEBILLOTTE-CUCHET Violaine, « Corps, sexualité et genre dans les mondes grec et romain ", DHA, suppl. 14, 2015, p. 83-108. Disponible en ligne sur $<$ www.cairn.info/revue-dialogues-d-histoire-ancienne-2015-Supplement14-page-83.htm>.

BRUIT-ZAIDMAN Louise \& SCHMITT-PANTEL Pauline, « L'historiographie du genre : état des lieux », dans V. Sebillotte-Cuchet \& N. Ernout (éd.), Problèmes du genre en Grèce ancienne, Paris, Publications de la Sorbonne, 2007, p. 27-48.

BRULÉ Pierre, La fille d'Athènes. La religion des filles à Athènes à l'époque classique. Mythe, culte et société, Besançon / Paris, Annales littéraires de l'université de Besançon, 1987.

CALAME Claude, Poétique des mythes dans la Grèce antique, Paris, Hachette, 2000.

CALAME Claude, «Sacrifice des filles d'Érechthée et autochtonie. Fondations étiologiques dans l'Athènes classique », Classics@, 7, 2011. Disponible en ligne sur <https://chs.harvard.edu/ classics7-claude-calame-sacrifice-des-filles-derechthee-et-autochtonie/>.

CASTIGLIONI Maria Paola, Cadmos-serpent en Illyrie : itinéraire d'un héros civilisateur, Pise, Éd. Plus, 2010.

CHANTRAINE Pierre, Dictionnaire étymologique de la langue grecque. Histoire des mots, Paris, Klincksieck, 1968-1980, nouvelle édition avec en supplément les Chroniques d'étymologie grecques, Paris, Klincksieck, 2009.

CHIRASSI COLOMBO Ileana, « Atene - il mito (e Nicole Loraux) », I Quaderni del Ramo d'Oro on-line, 3, 2010, p. 1-17. Disponible en ligne sur <www.qro.unisi.it/frontend/node/68>.

CHRISTOPOULOS Ménélas, « Poseidon Erechtheus and EPEX $\Theta \mathrm{HI} \Sigma \Theta \mathrm{A} \Lambda \mathrm{A} \Sigma \Sigma \mathrm{A}$ », dans R. Hägg (éd.), Ancient Greek Cult Practice from the Epigraphical Evidence, Stockholm, P. Åströms, 1994, p. 123-130.

DALADIER Nathalie, « Les mères aveugles », Nouvelle Revue de psychanalyse, 19, 1979, p. 229-244. 
DETIENNE Marcel, L'invention de la mythologie, Paris, Gallimard, 1981.

DETIENNE Marcel \& SISSA Giulia, La vie quotidienne des dieux grecs, Paris, Hachette, 1989.

DETIENNE Marcel, Comment être autochtone. Du pur Athénien au Français raciné, Paris, Seuil, coll. « Librairie du xx siècle », 2003.

GEORGOUDI Stella, « Gaia/Gê : entre mythe, culte et idéologie », dans S. des Bouvrie (éd.), Myth and Symbol I. Symbolic Phenomena in Ancient Greek Culture, Bergen, Norwegian Institute at Athens, 2002, p. 113-134.

GEORGOUDI Stella, « Lysimaché, la prêtresse », dans N. Loraux (éd.), La Grèce au féminin, Paris, Les Belles Lettres, 2003, p. 167-213.

GOURMELEN Laurent, Kékrops, le roi-serpent. Imaginaire athénien, représentations de l'humain et de l'animalité en Grèce ancienne, Paris, Les Belles Lettres, 2004.

HALL Jonathan M., Hellenicity: Between Ethnicity and Culture, Chicago / Londres, The University of Chicago Press, 2002.

HURST André, «Bâtir les murailles de Thèbes », dans P. A. Bernardini (éd.), Presenza e funzione della città di Tebe nella cultura greca (Atti del Convegno Internazionale, Urbino, 7-9 juillet 1997), Pise / Rome, 2000, p. 63-81.

JOUAN François \& VAN LOOY Herman, Euripide, tome VIII, $2^{\mathrm{e}}$ partie : Fragments, Paris, Les Belles Lettres, « Collection des Universités de France », 2000.

LÉTOUBLON Françoise, Fonder une cité, Grenoble, ELLUG, 1987.

LÉTOUBLON Françoise, «Le soleil et la lune, l'eau et le feu selon Meillet, de la grammaire comparée à l'anthropologie ", dans S. Auroux (éd.), Antoine Meillet et la linguistique de son temps, Histoire, Épistémologie, Langage (HEL), 10 (2), 1988, p. 127-139.

LÉTOUBLON Françoise, « Ilion battue des vents, Troie aux larges rues : la représentation de Troie dans l'Iliade ", dans M. Reddé (éd.), La naissance de la ville dans l'Antiquité, Paris, De Boccard, 2003, p. 27-44.

LORAUX Nicole, 1981, L'invention d'Athènes. Histoire de l'oraison funèbre dans la "cité classique ", Paris, Mouton / EHESS, 1981 (nouvelle édition abrégée et augmentée d'une préface sous le même titre, chez Payot, Paris, 1993).

LORAUX Nicole, Façons tragiques de tuer une femme, Paris, Hachette, coll. « Textes du xx siècle ", 1985 (nouveau tirage, 1992).

LORAUX Nicole, Les enfants d'Athéna. Idées athéniennes sur la citoyenneté et la division des sexes, réédition augmentée d'une postface, Paris, Points Seuil, 1990 (éd. originale La Découverte).

LORAUX Nicole, L'invention d'Athènes. Histoire de l'oraison funèbre dans la "cité classique ", nouvelle édition abrégée, augmentée d'une préface, Paris, Payot Rivages, 1993.

LORAUX Nicole, Né de la Terre. Mythe et politique à Athènes, Paris, Seuil, coll. « Librairie du $\mathrm{xx}^{\mathrm{e}}$ siècle », 1996.

LORAUX Nicole, La voix endeuillée. Essai sur la tragédie grecque, Paris, Gallimard, 1999.

LORAUX Nicole, « De quelques illustres inconnues ", introduction à N. Loraux (éd.), La Grèce au féminin (paru d'abord en italien, Bari, 1993), Paris, Les Belles Lettres, 2003, p. XI-XXXVI. 
MACÉ Arnaud, «L'autochtonie et la fécondité de la terre : l'imaginaire politique de la nation naturelle en Grèce ancienne », Millars. Espai i història, 44 (1), 2018, p. 17-43. Disponible en ligne sur <www.e-revistes.uji.es/index.php/millars/article/view/3437/2834>.

MEILLET Antoine, «Les noms du "feu" et de l'“eau" et la question du genre », MSL, 22, 1920, p. 249-256.

MEILLET Antoine, «Essai de chronologie des langues indo-européennes », Bulletin de la Société de linguistique de Paris, 32, 1931, p. 1-28.

MEILLET Antoine, «Linguistique et anthropologie », L’Anthropologie, 43, 1933, p. 41-46.

MEILLET Antoine, «Le genre féminin dans les langues indo-européennes », communication à la Société de psychologie du 14 juin 1923, Journal de psychologie, 943, 1951, p. 24-35.

PATTERSON Cynthia, « Hai Attikai: The Other Athenians », dans M. Skinner (éd.), Rescuing Creusa: New Methodological Approaches in Antiquity, Helios, ${ }^{\circ}$ spéc. 13 (2), 1987, p. 49-67.

PELLIZER Ezio, « L'anthropomorphisme des dieux dans la Grèce antique », dans R. Duits \& F. Quiviger (éd.), Images of the Pagan Gods. Papers of a Conference in Memory of Jean Seznec, Londres / Turin, The Warburg Institute / Nino Aragno Editore, coll. « Warburg Institute Colloquia », 14, 2009, pp. 267-280.

PELLIZER Ezio, « Introduzione. Mater lacrimosa. Costruzione dei modelli femminili del dolore in Grecia antica », Quaderni del Ramo d'Oro on-line, 3, 2010, p. I-X. Disponible en ligne sur $<$ www.qro.unisi.it/frontend/node/67>.

RICHIR Marc, La naissance des dieux, Paris, Hachette, 1995 (nouveau tirage, 1998).

SAINTILLAN David, « Du festin à l'échange : les grâces de Pandora », dans F. Blaise, P. Judet de La Combe \& P. Rousseau, Le Métier du mythe. Lectures d'Hésiode, Lille, Presses universitaires du Septentrion, 1996, p. 315-348.

SAUZEAU Pierre, Les partages d'Argos. Sur les pas des Danaïdes, Paris, Belin, 2005.

SEBILLOTTE-CUCHET Violaine, « Les antiquistes et le genre », dans V. Sebillotte-Cuchet \& N. Ernout (éd.), Problèmes du genre en Grèce ancienne, Paris, Publications de la Sorbonne, 2007a, p. 11-26.

SEBILLOTTE-CUCHET Violaine, « Une politique des genres, le serment des éphèbes athéniens », dans V. Sebillotte-Cuchet \& N. Ernout (éd.), Problèmes du genre en Grèce ancienne, Paris, Publications de la Sorbonne, 2007b, p. 233-245.

SEBILLOTTE-CUCHET Violaine, « Touchée par le féminisme. L'Antiquité avec les sciences humaines ", dans P. Payen \& E. Scheid-Tissinier (éd.), Anthropologie de l'Antiquité. Anciens objets, nouvelles approches, Turnhout, Brepols, 2012.

SEBILLOTTE-CUCHET Violaine, «Citoyens et citoyennes dans la cité grecque classique. Où joue le genre ?», Tempo, 21 (38), 2015. Disponible en ligne sur <www.scielo.br/scielo.php? pid=S1413-77042015000200281\&script=sci_arttext\&tlng=fr>.

SEBILLOTTE-CUCHET Violaine, «Ces citoyennes qui reconfigurent le politique. Trente ans de travaux sur l'Antiquité grecque », Clio, 43, 2016. Disponible en ligne sur <https://doi.org/10.4000/ clio.12998>.

VERNANT Jean-Pierre, « Le Dionysos masqué des Bacchantes d'Euripide », dans J.-P. Vernant \& P. Vidal-Naquet, Mythe et tragédie 2, Paris, La Découverte, 1986, p. 253-287.

VERNANT Jean-Pierre, Pandora, la première femme, Paris, Bayard, 2006. 
VIAN Francis, Les Origines de Thèbes. Cadmos et les Spartes, Paris, Belles Lettres, 1963.

VIDAL NAQUET Pierre, « Edipe entre deux cités. Essai sur l'đEdipe à Colone », dans J.-P. Vernant \& P. Vidal-Naquet, Mythe et tragédie 2, Paris, La Découverte, 1986, p. 175-211.

WATHELET Paul, « Le Pélion, $\pi \eta \lambda$ ó et Pélée », dans H. H. Hornung (éd.), Disputationes ad montium vocabula aliorumque nominum significationes pertinentes, Vienne, Verlag der Wiener Medizinischen Akademie, 1969, p. 511-516.

WATKINS Calvert, The American Heritage Dictionary of Indo-European Roots, Boston, Houghton Mifflin Harcourt, 2000.

ZEITLIN Froma I., « Thebes: Theater of Self and Society in Athenian Drama », dans F. I. Zeitlin \& J. J. Winkler (éd.), Nothing to Do with Dionysos? Athenian Drama and Its Social Context, Princeton, Princeton University Press, 1990, p. 130-167.

\section{NOTES}

1. La rencontre a été publiée dans Les nouveaux Cahiers franco-polonais, 4, 2005, p. 217-229.

2. La table ronde de Trieste en hommage à Nicole Loraux intitulée Donna, Mito, Miturgia a été publiée en 2010 dans le numéro 3 des Quaderni del Ramo d'Oro. Renvoyons particulièrement à l'introduction que lui a donnée Ezio Pellizer (2010) et à l'article d'Ileana Chirassi Colombo (2010). Ezio Pellizer a par ailleurs lui-même évoqué la question de l'autochtonie dans un bel article sur l'anthropomorphisme des dieux grecs, en citant Né de la terre et en reproduisant le stamnos de Vulci, cf. Pellizer (2009, 272-273 et fig. 5, 279).

3. D'autant que nous nous sommes rencontrés par l'intermédiaire de Nicole Loraux. Jean Alaux avait déjà travaillé sur la question de l'autochtonie, voir Alaux (1997a et 1997b).

4. À dire vrai, le livre de Detienne, plutôt que de discuter les arguments de Loraux, les résume, avec le même mordant que celui qu'il applique aux versions mythologiques discutées. Le véritable débat semble avoir eu lieu avec l'article bien argumenté de Cynthia Patterson dans Rescuing Creusa (1987), qui repose sur une analyse linguistique approfondie des textes pour montrer que, si l'adjectif féminin Athenaia ne se rencontre pas à propos des femmes d'Athènes, elles sont pourtant désignées par l'adjectif Attikai et comme astai, non pas exclues de la cité. Selon elle, Loraux et Vidal-Naquet ont cherché une validation mythologique de leur vue au lieu d'étudier en détail l'usage athénien. Voir aussi, dans le droit-fil du courant féministe, SebillotteCuchet (2012, 155-159), qui analyse la différence des sexes dans la pensée de Nicole Loraux comme «structure psychique et politique » (titre de son point 2.2).

5. Sur la difficulté grecque à mythologiser l'origine et la fondation, et à opérer des synthèses entre traditions variées, voir Richir (1995, 61-67).

6. Le corpus de travail sera celui de Nicole Loraux: Hésiode et les mythographes (Apollodore, Antoninus Liberalis, etc.), ainsi que le théâtre classique et l'oraison funèbre.

7. Patterson $(1987,57-63)$ : « Augustine, City of God, 18.9: An Athenian Foundation Myth?».

8. Voir aussi Sebillotte-Cuchet (2015 et 2016).

9. Parmi la floraison de textes produits sur le genre, nous avons cité ceux qui s'intéressent aux mythes d'autochtonie, mais les lecteurs qui voudraient s'informer plus largement sur la question pourront lire Sebillotte-Cuchet (2007a), Bruit-Zaidman \& Schmitt-Pantel (2007), Boehringer \& Sebillotte-Cuchet (2013 et 2015), liste qui ne prétend pas être exhaustive. Le rôle de Nicole Loraux dans les études féministes apparaît bien dans l'ensemble de ces études.

10. Loraux (1996, 9-26). Voir aussi Georgoudi (2002, 113-134).

11. Hésiode, Théogonie, 570-612; Les Travaux et les Jours, 59-105. Voir en particulier Théogonie, 571

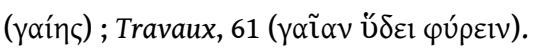


12. Prométhée auquel une tradition postérieure attribuera d'ailleurs, par modelage de la terre, la création du genre humain dans son ensemble. Sur l'échange des femmes limité au rapport des hommes entre eux, excluant toute relation de l'homme à la femme, voir Saintillan (1996) et Vernant (2006).

13. Dans les Travaux, le mythe de Pandora est suivi par celui des cinq races créées par les Olympiens.

14. Première esquisse d'ordre spatialisé : Gaia enfante Ciel, les montagnes (et donc les vallons), Flot (Pontos).

15. Deuxième esquisse d'ordre spatialisé, plus complexe, par les Titans, mais Ouranos bloque la génération et étouffe l'espace.

16. Hésiode, Théogonie, 665-819.

17. Loraux $(1996,14)$.

18. Apollodore, Bibliothèque, I, 7, 2 ; cf. fragments 2, 4, 6, 234 d'Hésiode (R. Merkelbach \& M. L. West (éd.), Fragmenta Hesiodea, Oxford, Clarendon Press, 1967); Akousilaos, FGrHist, 34-35; Pindare, Olympique, IX, 41 s., voir les premiers chapitres de Hall (2002, 56-89, en particulier sur Hellen et ses fils; chap. III sous-titré «Blood and Belonging in Early Greece »). Voir aussi Macé $(2018,21$, n. 10).

19. Mélia, sœur d'Inachos (Gantz, 2004, 352), porte le nom du frêne comme les nymphes Méliai. C'est la seule étymologie possible pour son nom, Chantraine le confirme.

20. Voir Richir (1995, 45-48), qui souligne que la refondation mythique de l'humanité emprunte ici « le plus long détour » possible. Voir plus largement Sauzeau (2005).

21. Loraux (1996, 72-74).

22. Homonyme d'un autre Pélasgos, Argien, fils de Triopas, oncle d'Io et descendant de Phoroneus. Signalons que les Suppliantes d'Eschyle mettent en scène un roi d'Argos, Pélasgos, fils de l'autochtone Palaichthôn (Eschyle, Suppliantes, 249-251) dont on sait que la lignée sera remplacée par celle des descendants de Lyncée et d'Hypermestre, elle-même épigone d'Io et donc issue de la lignée de Phoroneus.

23. Difficulté des commencements, de la première naissance humaine, comme s'il n'allait pas de soi, pour un Grec, de penser l'absolu de l'origine et de l'articuler au phénomène de répétition des générations successives, surtout lorsque, dans le cas d'Athènes, l'on rappelle à l'envi que tout citoyen est né du sol, alors qu'il se sait aussi issu de l'union d'un homme et d'une femme. À moins de considérer comme le fait $\mathrm{C}$. Calame dans sa réflexion sur la géopolitique des mythes liés à la descendance d'Iô (2000, 117-144), référés aux mythes d'origine proprement athéniens, qu'une cohérence globale peut être dégagée d'une reconstruction de l'ensemble : «L'établissement de l'ordre civique et religieux dans l'affirmation de l'autochtonie passe [...] par trois étapes distinctes : au surgissement asexué du premier roi de la cité hors de son sol même succède la naissance de cette même terre d'un enfant procréé dans une fécondation différée, avant que ne naissent d'une relation sexuelle accomplie des filles dont le sang est en partie destiné, par le sacrifice, à retourner dans le sol originaire " (Calame, 2000,134); on aura reconnu Cécrops, Érichthonios, et enfin Érechthée, Praxithéa et leurs filles. Les Suppliantes d'Eschyle auraient présenté au public athénien une version dramatisée du même processus, transféré à Argos (Pélasgos, fils de Palaichthôn ; attouchement d'Io par Zeus ; sacrifice des fils d'Égyptos conduisant enfin à l'union sexuée entre mortels). Cf. ibidem, p. 135 : «Tout se passe comme si les légendes de l'autochtonie présentaient un déficit qui devait être comblé par un acte de refondation de la cité, à partir de l'extérieur, dans un mouvement qui rappelle celui des récits de fondation des cités coloniales!»

24. Wathelet (1969).

25. Pour une vue d'ensemble, voir Richir (1995, 43-58). Sur le mythe thébain, voir aussi Vian (1963). 
26. De la descendance d'Europe, unie à Zeus et mère de Minos et de son ou ses frères (Rhadamanthe et Sarpédon, non constamment réputé fils d'Europe), procède la lignée royale de Crète.

27. Tandis que sa sœur Antiope, unie à Zeus, engendrera les jumeaux Amphion et Zéthos. Il est vrai que dès l'odyssée, une tradition attribue aux deux frères la fondation de la cité (Homère,

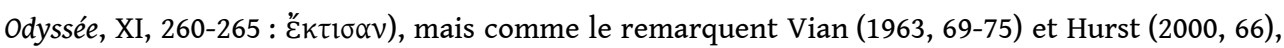
ils se situent à un autre niveau : leur geste essentiel consiste à entourer la cité de remparts.

28. Castiglioni (2010).

29. Pindare, Olympique, I, 24.

30. On comprend pourquoi Platon, dans sa sourde polémique contre la cité athénienne et donc contre son propre mythe d'autochtonie, fait référence à ce paradigme «sidonien" ou "phénicien » pour étayer le «bel » et «utile mensonge » qui favorisera fraternité et unanimité entre les citoyens, même si une opération seconde s'impose pour légitimer la hiérarchie des trois classes d'hommes: République, III, $414 \mathrm{c}$-e (avantages de la croyance autochtone: les citoyens regarderont la terre comme leur "mère " et se regarderont comme des "frères »); Lois, II, $663 \mathrm{~d}-664$ a. Il s'agira alors de montrer à quel point le même est travaillé par l'autre (Loraux, 1996, 100-101, 176-178). Notons que Detienne (1981, 177-178) citait déjà cette critique platonicienne du « mythologème » d'autochtonie.

31. Voir les références aux travaux de Meillet qui vont de sa thèse sur les langues slaves en 1897 à 1936 dans Létoublon (1988). Cf. aussi Meillet (1933 et 1951).

32. Meillet $(1931,7)$.

33. C. Watkins (2000), chapitre «Indo-European and the Indo-Europeans »; Chantraine (2009, s. v. $\chi \theta \omega \dot{v})$.

34. On devrait plutôt dire que la terre, en grec du moins, ne se dit jamais au masculin.

35. Pour une vue d'ensemble, voir les tableaux établis par Brulé (1987, 14-15). On connaît par Antoninus Liberalis, Métamorphoses, VI, un certain Périphas qui vécut en Attique avant même Cécrops: bon roi, honoré à l'égal de Zeus, il suscita la jalousie du père des dieux, qui le transforma en aigle et lui attribua la royauté sur les oiseaux (cf. Ovide, Métamorphoses, VII, 399-400 : «Palladas arces, quae te, iustissima Phene, / Teque, senex Peripha, pariter uidere uolantes »). Sur le mythe de Périphas, voir Richir $(1995,3)$.

36. Sur lequel voir Gourmelen (2004) et notre compte rendu dans Gaia (Alaux, 2006).

37. Detienne \& Sissa $(1989,234)$.

38. Saint Augustin, Cité de Dieu, 18, 9.

39. Le nom de la laine en grec est عĩ attique Épıv, ce qui explique peut-être les jeux de mots sur les noms des fondateurs (voir ci-dessous chez Aristophane).

40. À ne pas confondre avec son homonyme troyen, fils de Dardanos, ancêtre de Priam: Létoublon $(1987,81)$; Létoublon (2003, 31-32).

41. Homère, dans Iliade, II, 547-549, implique un Érechthée autochtone.

42. Selon Brulé $(1987,18)$, Érechthée serait une figuration plus "politique», et Érichthonios correspondrait à la figure de l'« enfant divin».

43. Apollodore, Bibliothèque, III, 14,6: Cranaos authochtone, contemporain du déluge et de Deucalion, père d'Atthis, qui donna son nom à l'Attique.

44. Sur Cranaos, roi d'Athènes, voir Eschyle, Euménides, 1011 ; Aristophane, Oiseaux, 122-123 :

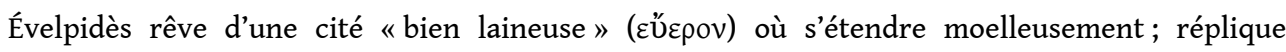
d'Épops (Térée) : «Mais alors, tu cherches une ville plus grande que celle des Cranaens ?»; le jeu se fait à la fois sur l'antonymie (douceur / dureté) et sur l'homonymie entre le nom de Cranaos et

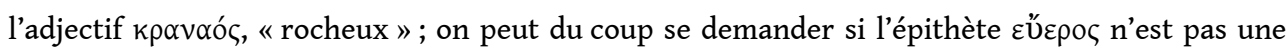
allusion indirecte à l'autochtonie fondatrice d'Athènes, à ce brin de laine ('ćprov, voir ci-dessus, n. 39) qui féconda le sol de l'Acropole ; Apollodore rapporte que l'on dérive le nom d'Érichthonios 
soit d'ع́pı « querelle », soit d'ع́prov « brin de laine »; voir Brulé $(1987,14)$, qui relie la première hypothèse à la version d'une «lutte » entre Athéna et Héphaïstos, la seconde à la fuite de la déesse : ibidem (p. 141, n. 30 : l'étymologie la plus probable de la première partie du composé reposerait sur le préverbe intensif $\dot{\varepsilon} \rho \mathrm{l}-)$.

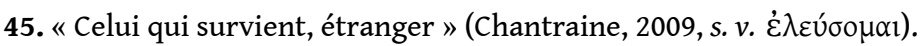

46. Sur ces textes et sur la représentation d'elle-même que s'y donne la cité, voir Loraux (1993). L'oraison funèbre ironique du Ménexène de Platon, prononcée par une femme (Aspasie), pousse au plus haut point la logique des topoi qu'elle pastiche : voir par exemple la «bonne naissance»

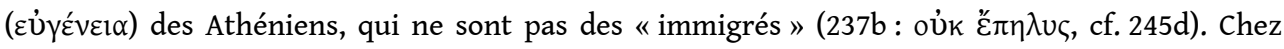

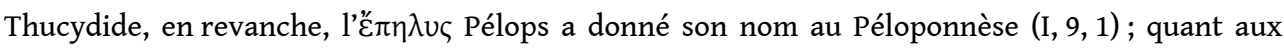
Spartiates, ils se flattent, en somme, de venir d'ailleurs (cf. Detienne, 2003, 67).

47. Le Ménexène met en rapport l'origine bigarrée des autres cités et l'inégalité de leurs gouvernements: "Les autres cités sont constituées de populations de toute provenance, et formées d'éléments inégaux, d'où résulte chez elles l'inégalité des gouvernements, tyrannies et oligarchies. » (238e, 2-4) Chez les Athéniens, au contraire, "l'égalité d'origine (iøoyoví $\alpha$ ), établie par la nature, nous oblige à rechercher l'égalité politique (iøovouí $\alpha$ ) établie par la loi, et à ne céder le pas aux autres qu'au nom d'un seul droit, la réputation de valeur ( $\left.\alpha_{\rho \varepsilon \tau} \tilde{\varsigma} \varsigma\right)$ et de sagesse »(239a).

48. Sur ce point, voir Loraux $(1990,75-117)$.

49. Hydrie du peintre d'Oinanthé (Londres : $2^{\mathrm{e}}$ quart du $\mathrm{v}^{\mathrm{e}}$ siècle) : Loraux $(1990$, fig. 3$)$; Stamnos de Munich (2e quart du $v^{\mathrm{e}}$ siècle) : Brulé $(1987,46)$ et Loraux $(1990$, fig. 4) ; coupe de Kodros (Berlin : aux environs de 440) : Brulé $(1987,54)$, Loraux (1990, fig. 5a et 5b) ; Reliefs de Berlin, du Louvre et du Vatican : Brulé (1987, 52 et 54). Voir aussi Pellizer (2009).

50. Nous n'entrerons pas dans le débat sur la légitimité d'un parallélisme entre la scène mythique et le rituel des Amphidromies à Athènes, cf. Brulé (1987, 57-62), Loraux (1990, 263-264) : l'essentiel demeure à nos yeux la dissociation entre la maternité biologique (assumée par Gê) et l'éducation de l'enfant confiée à une figure nourricière placée traditionnellement du côté masculin (voir Daladier, 1979).

51. Voir Vidal-Naquet (1986) ; Zeitlin (1990).

52. Loraux $(1996,90)$.

53. Eschyle, Sept contre Thèbes, 938-940 (notre traduction). Que, dans d'autres passages, le texte d'Eschyle se refuse apparemment à jouer sur l'opposition entre le sang versé ( hérité ( $\alpha \tilde{i} \mu \alpha)$, mais associe les deux termes qui dès lors se renforcent, importe peu (Sept contre Thèbes, 44-45; 737 : il y aurait d'ailleurs matière à discussion et l'occasion serait belle de se demander si l'ironie tragique n'est pas justement à l'œuvre dans les deux passages cités) :

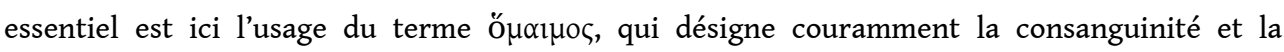
fraternité de sang.

54. Vernant (1986).

55. Euripide, Bacchantes, 1-42 ; 86-104.

56. Euripide, Bacchantes, 213, 229, 265, 507, 539-541, 995-996, 1015-1016, 1025-1026, 1030, 110, $\underline{1155}, \underline{1274}$ (les occurrences soulignées sont celles où, par une intéressante condensation, c'est Penthée et non son père qui est « né du dragon » ou « né de la terre » (үฑүદvńৎ); aux v. 1330-1332 et 1357-1358, l'image du dragon ne s'applique plus qu'à la métamorphose régressive de Cadmos et d'Harmonie. Sur Cadmos-serpent, voir Castiglioni (2010).

57. Detienne \& Sissa (1989, 231-252), cf. la réponse de Loraux (1990, 266-269) ; Detienne (2003, 19-120).

58. Jouan \& Van Looy $(2000,95-132)$.

59. Sur les différentes versions de leur suicide, voir Brulé $(1987,28-31)$ : elles ont ouvert la ciste contenant Érichthonios; elles ont fait vœu de ne pas survivre à leur sœur sacrifiée lors de la 
guerre contre Eumolpos. N. Loraux a souligné l'ironie tragique qui probablement préside aux paroles d'Athéna, lorsqu'elle prescrit d'enterrer Chronia sous le même tertre que ses sœurs «à cause de leur noblesse » (fr. 68-70), alors même qu'elles se sont suicidées (Loraux, 1985, 80-81). Sur la tirade de Praxithéa, le mythe et l'autochtonie, voir aussi Calame (2011).

60. D'où viendront plus tard la réconciliation du dieu et du roi et le culte de Poséidon-Érechthée qu'il faut sans doute mettre en rapport avec la volonté athénienne de souveraineté à la fois terrestre et maritime (voir Brulé, 1987, 27 ; Christopoulos, 1994).

61. Georgoudi (2003, notamment 176-178 sur le nom de Praxithéa et sur son rapport avec Athéna). Le rôle souvent éminent des prêtresses d'Athéna Polias ne saurait en aucun cas préjuger d'une quelconque importance politique des femmes sui generis (Loraux, 2003, XXIII-XXIV).

62. Chantraine (2009, s. v. ö $\rho \mu \alpha)$.

63. Cf. Loraux $(1990,267)$.

64. Elle est la fille du dieu-fleuve Céphise, ce qui n'est pas la même chose. Une femme peut cependant transmettre l'autochtonie sans être elle-même autochtone ni " jouir de l'autochtonie » (Brulé, 1987, 395) : c'est le cas de Créuse, fille d'Érechthée, épouse de Xouthos, mais d'abord unie à Apollon, dont elle eut l'enfant Ion, promis à régner à Athènes et à perpétuer la lignée d'Érechthée (voir Loraux, 1990, 197-253 ; 264-266 sur l'Ion d'Euripide ; Brulé, 1987, 23-24).

65. Loraux (1993, 45-47).

66. Loraux (1999).

67. Le mouvement inverse, mais toujours critique, s'observe dans la scène des Suppliantes d'Euripide, véritable "réflexion en acte sur l'oraison funèbre " (voir Loraux, 1993, 69-71), où Thésée vient canaliser et orienter vers l'éloge la plainte entamée par Adraste et les mères sur les corps des guerriers argiens morts dans la guerre contre Thèbes (v. 799 et suiv.).

68. Loraux (1996, 130-134). Les sonorités "galactiques» sont une référence de l'auteur à J. Derrida (Glas, Paris, Galilée, 1974) : le signifiant $\mu \varepsilon \mu$ í $\mu \eta \tau \alpha$ est le seul à y échapper dans la phrase citée (p. 142). Voir déjà Loraux $(1990,13-14)$ à propos de ce passage : « [...] le signifiant détruit le signifié (et, dérivée dans le signifié, la maternité des femmes prête en réalité son langage à l'énoncé). " Voir aussi Patterson (1987, 56-57), qui propose une explication alternative: "The woman of Athens is an imitation of the Attic Earth and by virtue of her imitation partakes of the honor and venerability of Mother Attike.[...] Attike and aste are words which not only reveal the fact of women's membership in the Athenian polis but show as well the generally non-political, non-abstract, "earthy" character of that membership. "

69. Loraux $(1996,31,97)$.

70. Loraux (1996, 31-34).

71. Loraux $(1990,15,21)$.

72. À moins de rester dans le registre du «comme » et de ne pas intervertir comparant et comparé : «La terre dormait nue et tourmentée comme une mère dont la couverture aurait glissé. » (A. Platonov, cité par Pierre Michon, La grande Beune, Paris, Verdier, 1996)

\section{RÉSUMÉS}

En hommage à Ezio Pellizer qui admirait Nicole Loraux, nous revenons ici sur la question des mythes grecs d'autochtonie et le paradoxe selon lequel les Athéniens considèrent la terre comme leur mère et sont les seuls des Grecs à avoir donné à leur cité le nom d'une déesse (Athéna). 
Suivant Nicole Loraux, le mythe d'autochtonie est l'outil idéologique qui a permis aux Athéniens d'exclure les femmes de la citoyenneté.

As a tribute to Ezio Pellizer who admired Nicole Loraux, we return to the issue of Greek myths of autochtony, and the paradoxical attitude of the Athenians, who considered the soil as their mother and gave their city the name of a goddess (Athena), the only Greeks to do so. For Nicole Loraux, the myth of autochtony is the ideological tool that allowed the Athenians to exclude women from citizenship.

INDEX

Keywords : myths, autochthony, earth, gender, origin of mankind

Mots-clés : mythes, autochtonie, terre, genre, origine des hommes

\section{AUTEURS}

JEAN ALAUX

Université Rennes 2

alaux.jean@numericable.fr

\section{FRANÇOISE LÉTOUBLON}

Université Grenoble Alpes

francoise.letoublon@univ-grenoble-alpes.fr 